\begin{tabular}{ccc}
\hline International Journal of Engineering \& Technology, $7(4.30)(2018) 269-270$ \\
SPC \\
Website w ww. sciencepubco.com/index.php/IJET \\
Research paper
\end{tabular}

\title{
Optical Absorption of Plasmonic Cylindrical Gold Nanoparticle in Hexagonal Geometry
}

\author{
Norasikin M Nasar ${ }^{1}$, Rosmila Abdul-Kahar ${ }^{1 *}$, Nor Shamsidah Amir Hamzah ${ }^{1}$, Fahmiruddin Esa ${ }^{1}$ \\ ${ }^{1}$ Faculty of Applied Sciences and Technology, Universiti Tun Hussein Onn Malaysia, \\ Educational Hub Pagoh, 84000 Muar, Johor, Malaysia. \\ *Corresponding author E-mail: rosmila@uthm.edu.my
}

\begin{abstract}
A high quality solar cell depends on how good the design of the solar cell can absorb light. In this study, cylindrical gold nanoparticles were embedded into indium tin oxide (ITO) layer and silicon layer arranged in hexagonal geometry on plasmonic solar cell simulation design. The aim is to investigate the optical absorption percentage in terms of wavelength and angle of incidence for the solar cell design. The numerical results showed that the highest absorption has occurred in $480 \mathrm{~nm}$ in the range of visible spectrum. In this wavelength, the highest absorption occurred at the incidence angle of 48 degree.
\end{abstract}

Keywords: Gold Nanoparticle, Optical Absorption, Plasmonic Nanostructure, Numerical Simulation, Solar Cell

\section{Introduction}

Many solar applications arising in connection with plasmonic that use metal nanoparticles like gold and silver [1-3] is the result of the unique properties of surface plasmon [1]. These unique properties include manipulation and transport of light at nanometer scale due to their highly localized electromagnetic fields [4]. When using the surface plasmon properties, geometrical dimensions of the nanostructure can be controlled. It is possible to enhance the optical absorption because the light could enter the structure with various angles of incidence at certain wavelengths [5-6]. However, there are still exists the limited absorption of light effect at geometry structure for optical absorption efficiency. Recently, several numerical simulations have been investigated on metal nanostructures [7]. Simulations are used as a method to understand fundamental of plasmonic and investigated the effective factors of plasmon properties on nanoparticles. Finally, more plasmonic device can be designed to become a commercial product in industry [7]. Simulation of plasmons in nanostructure usually used as a method to solved the Maxwell's equations for nanoparticles with several geometry, size and dielectric function [7]. In this paper, nanoscale plasmonic structures are explored for performance improvement. These structures used as a ways to guide and trap light for absorption and the overall performance in solar applications and optical properties [8]. Plasmonic structure in hexagonal geometry of cylindrical nanoparticle gold is modelled using COMSOL Multiphysics in electromagnetic wave propagation of frequency domain.

\section{Theory and methodology}

The interaction of light with $\mathrm{Au}$ nanostructures simulations were carried out with COMSOL Multiphysics which applies Finite Element Method to discretize Maxwell's equations as follows:

$$
\nabla \times E=-\mu \partial H / \partial t
$$

$\nabla \times H=\varepsilon \partial E / \partial t+\sigma E$

where, electric field $E$, magnetic field $H$, permeability $\mu$, permittivity $\varepsilon$, and conductivity $\sigma$ [9]. Maxwell's equations are indispensable to accurately predicting the interaction of matter in nanostructures as full-wave electromagnetic solvers [10]. Absorption were calculated using Beer-Lambert Laws that expressed as

$A=\log _{10}\left(I / I_{o}\right)$

where $A$ is absorption, $I$ is the intensities of incident light and $I_{0}$ is transmitted light after passing through the structure [11].

\section{Simulation Model}

The design of plasmonic gold nanoparticles in hexagonal geometry structure is adapted from [8] as shown in Figure 1 (a) and Figure 1 (b). The cylindrical gold nanoparticles of the hexagonal geometry structure have height, $80 \mathrm{~nm}$ and the radius, $20 \mathrm{~nm}$. The thickness of $\mathrm{Al}$ and ITO layers are taken as $80 \mathrm{~nm}$ and $20 \mathrm{~nm}$ while the silicon layer thickness is $300 \mathrm{~nm}$. The width, W and length, L of the structure are equal which is $280 \mathrm{~nm}$. Finite Element Method (FEM) was used in this work to analyses the absorption on plasmonic structure in hexagonal geometry of cylindrical nanoparticle gold. The absorption was calculated in the wavelength range 100 $\mathrm{nm}-1000 \mathrm{~nm}$. 


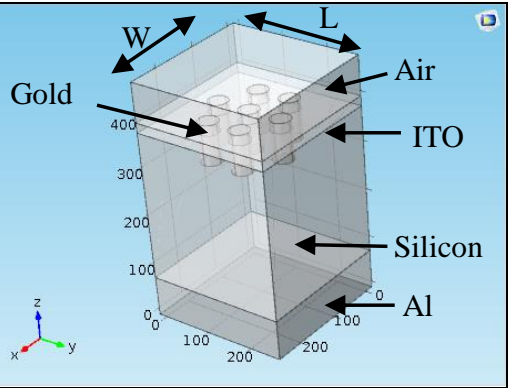

(a)

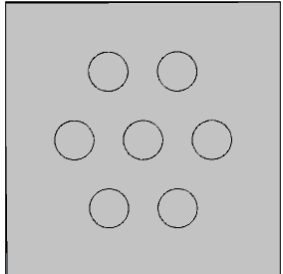

(b)
Fig. 1: (a) Schematic of the plasmonic gold nanoparticle in hexagonal geometry structure (b) 2D hexagonal geometry of gold nanoparticle viewed from top.

\section{Results and Discussion}

In this section, the simulation results were shown in terms of absorption versus of wavelength and angle of incident. The wavelengths used were in the range of 100 to $1000 \mathrm{~nm}$ while the angles of incident were taken in the range of 0 degree to 90 degree.

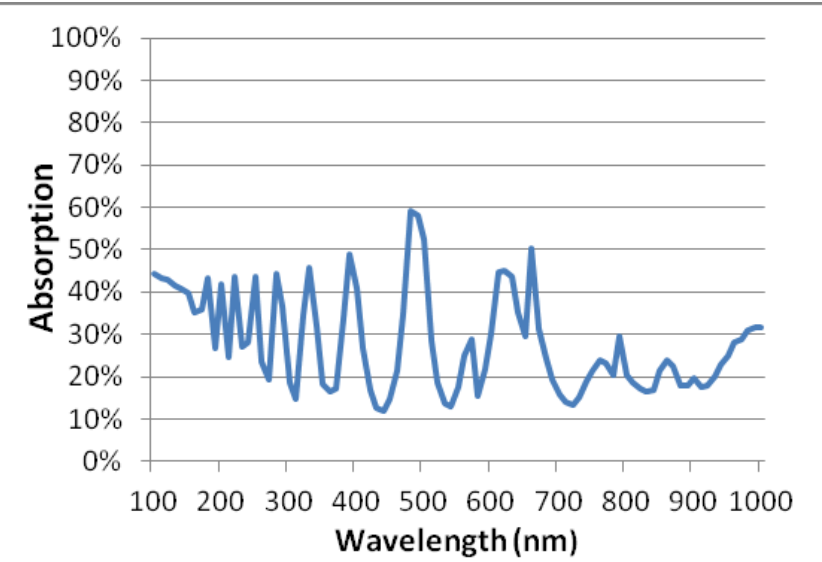

Fig. 2: Absorption of cylindrical gold nanoparticles with different wavelength.

Figure 2 shows the absorption with different wavelength of plasmonic gold nanoparticle in hexagonal geometry structure. The maximum absorption is $59 \%$ at a wavelength of $480 \mathrm{~nm}$ which is the highest peaks value in absorption. The second highest peaks happened at $660 \mathrm{~nm}$ at about $50 \%$. This highest peak occurs in frequency range of visible spectrum from $400 \mathrm{~nm}$ to $800 \mathrm{~nm}$ which give benefit to improve absorption and trapping the light in solar application. If compared to the result of Aluminium nanoparticle in [8], the maximum absorption rate is too low. Aluminium performed much better than gold with the same geometry. Further study need to be done in term of the arrangement of distance between cylindrical gold nanoparticles itself and also with different radius and height of cylindrical gold nanoparticles.

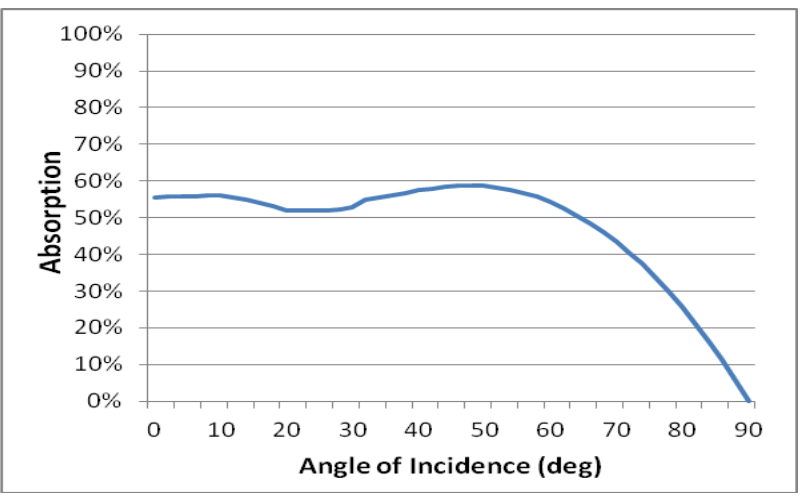

Fig. 3: Absorption as a function of incidence angle in $480 \mathrm{~nm}$
Wavelength at $480 \mathrm{~nm}$ was taken to analyses due to their highest peaks value in absorption as shown in Figure 2. The effect of absorption in the wavelength of $480 \mathrm{~nm}$ was showed in Figure 3 with different angle of incidence of optical light. The simulation results started with absorption value at about $56 \%$ when light directly on top of the surface at 0 degree. The value of absorption is high until it achieves a maximum absorption at 48 degree which about 59\% and then it started to decreased at 50 degree until 90 degree. These means that there are exist the high limits as a function of angle of incidence of optical light for the absorption.

\section{Conclusion}

This paper has presented the absorption of light based on plasmonic gold nanoparticle in hexagonal geometry as function of wavelength and angle of incidence. Results showed that the highest absorption has occurred in the range of visible spectrum. Surface plasmonic proved their efficiency using gold nanoparticle in hexagonal geometry in trapping more light or photon in solar applications although the absorption rate is lower than aluminium nanoparticles.

\section{Acknowledgement}

This research work was fully sponsored by Universiti Tun Hussein Onn Malaysia under GPPS 2017 vot U973 and TIER 1 phase 1/2017 vot U901. We extend our special thanks to I-Math Sdn Bhd in providing the Comsol Multiphysics 5.3a software trial for the simulation.

\section{References}

[1] Yue W, Yao P, Luo H \& Liu W (2017) Surface plasmon polariton nanocavity with ultrasmall mode volume. Journal of Physics D Applied Physics 50, 315102.

[2] Chou Chau YF (2017) Investigation of plasmonic effects on the metal nanoparticle arrays for biosensor applications. IOP Conference Series: Materials Science and Engineering 191, 012016.

[3] Mahmood AI, Ibrahim RK, Mahmood AI \& Ibrahim ZK (2018) Design and Simulation of Surface Plasmon Resonance Sensors for Environmental Monitoring. Journal of Physics: Conference Series 1003,012118

[4] Andrawis RR, Swillam MA \& Soliman EA (2014) Submicron omega-shaped plasmonic polarization rotator. Journal of Optics 16, 105001.

[5] Foroutan S, Dizaji HZ \& Riahi A (2017) Plasmon resonanceenhanced photocathode by light trapping in periodic concentric circular nanocavities on gold surface. Optik-International Journal for Light and Electron Optics 138, 223-228.

[6] Lee KY, Park SJ, Kim DH \& Kim YJ (2014) Effect of light incidence angle on optical absorption characteristics of low bandgap polymer-based bulk heterojunction organic solar cells. Japanese Journal of Applied Physics 53, 8S2.

[7] Ziashahabi A \& Poursalehi R (2015) The Effects of Surface Oxidation and Interparticle Coupling on Surface Plasmon Resonance Properties of Aluminum Nanoparticles as a UV Plasmonic Material. Procedia Materials Science 11, 434-437.

[8] Muhammad MH, Hameed MFO \& Obayya SSA (2014) Absorption Enhancement in Hexagonal Plasmonic Solar Cell. Numerical Simulation of Optoelectronic Devices (NUSOD), 63-64.

[9] Tang X, Zhou L, Du X \& Yang Y (2017) Enhancing absorption properties of composite nanosphere and nanowire arrays by localized surface plasmon resonance shift. Results in Physics 7, 87-94.

[10] He S, Sha WEI, Jiang L \& Choy WCH (2011) Finite Element Based Generalized Impedance Boundary Condition for Modeling Plasmonic Nanostructures. IEEE Transactions on Nanotechnology 11(2), 336-345.

[11] Hu M, Chen J, Li ZY, Au L, Hartland GV, Li X, Marquez M \& Xia Y (2006), Gold nanostructures: engineering their plasmonic properties for biomedical applications. Chemical Society Reviews 35, 1084-1094. 